in vivo $32: 1025-1032(2018)$

doi:10.21873/invivo.11342

\title{
Ultrasound Microbubble Delivery Targeting Intraplaque Neovascularization Inhibits Atherosclerotic Plaque in an APOE-deficient Mouse Model
}

\author{
HONG YUAN ${ }^{1}$, HAIQIANG HU ${ }^{1}$, JINDONG SUN $^{1}$, MINGJUAN SHI $^{1}$, HUAMIN YU $^{1}$, \\ CAIRONG LI $^{2}$, YU SUN ${ }^{3}$, ZHIJIAN YANG ${ }^{3,4}$ and ROBERT M. HOFFMAN ${ }^{4}$ \\ ${ }^{1}$ Yuhang District First People's Hospital, Hangzhou, P.R. China; \\ ${ }^{2}$ Medical College of Hangzhou Normal University, Hangzhou, P.R. China; \\ ${ }^{3}$ Origin Biosciences Inc., Nanjing, P.R. China; \\ ${ }^{4}$ AntiCancer, Inc., San Diego, CA, U.S.A.
}

\begin{abstract}
Background/Aim: Intraplaque neovascularization is often associated with plaque formation, development and instability, and clinical symptoms in atherosclerosis. The aim of the present study was to investigate a new strategy for treating athrosclerosis by ultrasound-targeted microbubble delivery (UTMD) targeting intraplaque neovascularization in an APOE-deficient mouse model of atherosclerosis. Materials and Methods: A mouse model of atherosclerosis was induced by feeding Apoe ${ }^{-/-}$mice a hypercholesterolemic diet and was verified with hematoxylin and eosin staining and intercellular adhesion molecule 1 (ICAM-1) expression. Targeted microbubbles (MB) were prepared by conjugating microbubbles with biotinylated antibody to ICAM1 (MBi) or with both biotinylated anti-ICAMI and the angiogenesis inhibitor Endostar (MBie). The targeted microbubbles were analyzed with epifluorescence microscopy and flow cytometry. The animals with induced atherosclerotic plaques received $M B i$ or MBie followed by UTMD treatment. Endostar treatment alone was given to other animals for comparison. Morphological assessment of atherosclerotic plaques was performed after treatment. The expression of angiogenesis marker CD31 was detected by immunohistochemical analysis. Results: Atherosclerotic plaques developed in the entire aorta
\end{abstract}

This article is freely accessible online.

*These Authors are the co-first authors of this article.

Correspondence to: Hong Yuan, Yuhang District First People's Hospital, Hangzhou, Zhejiang, P.R. China. Tel: +86 57189369176, e-mail: yuanhongy@163.com

Key Words: Ultrasound, targeted microbubble, intraplaque neovascularization, atherosclerosis. with significant intraplaque ICAM-1 expression in the APOEdeficient mice following a 30-week hypercholesterolemic diet. Microbubbles were successfully conjugated with anti-ICAM-1 and Endostar, with a conjugation rate of $98.3 \%$ and $63.5 \%$, respectively. UTMD with MBie significantly reduced the area of atherosclerotic plaque as compared to the model control $(p<0.05)$. Treatment with Endostar and UTMD with MBie significantly reduced CD31 expression compared with the model control group $(p<0.01)$. Greater significant inhibitory effect on CD31 expression was found in the group treated with UTMD and MBie compared to the Endostar-and UTMD with MBi groups $(p<0.01)$. Conclusion: UTMD targeting intraplaque neovascularization was found to inhibit atherosclerotic plaque in a mouse model of atherosclerosis, suggesting the potential of microbubble-mediated ultrasound technology in aiding drug delivery for atherosclerosis treatment.

Atherosclerosis is a lipid-driven inflammatory disease that occurs in the intima of the arterial vessel wall and typically leads to the formation of atherosclerotic plaques (1). Unstable plaques are vulnerable and prone to rupture, which may cause occlusive luminal thrombosis and its consequences, including myocardial infarction, unstable angina, sudden cardiac death, and stroke (2). Accumulating evidence has linked plaque angiogenesis with plaque formation, development and instability, and clinical symptoms (3-6). Increased neovascularization has been detected in ruptured human aortic plaques and is frequently associated with pathological signs of plaque vulnerability (7-9). Therefore, plaque neovascularization represents an interesting therapeutic target for inhibiting progression of atherosclerosis and stabilizing lesions. It was reported that bevacizumab, a monoclonal antibody against vascular endothelial growth factor, inhibited intraplaque neovascularization with smaller atherosclerotic lesions as a result (10). Some studies have shown that the 
lipid-lowering drug atorvastatin inhibits the formation and development of neovascularization in human carotid plaques $(11,12)$. These findings have stimulated the search for potential treatments with potent angiogenesis inhibitors in order to reduce plaque neovascularization and progression.

Therapeutic ultrasound using ultrasound-targeted microbubble delivery (UTMD) has demonstrated great potential as a tool for targeted disruption of tumor vasculature $(13,14)$. UTMD is a process in which acoustic energy induces bubble collapse, generating microjets that create transient pores in the cell membrane and facilitating transport of large molecules into the cell cytoplasm (15-17). An intravascularlyadministered microbubble can deliver DNA plasmids, siRNAs, proteins, or conventional drugs to the cells of the blood vessel wall if appropriately activated by ultrasound (13).

In the present study, we prepared drug-loaded microbubbles targeting intraplaque neovascularization by conjugating microbubbles with an angiogenesis inhibitor Endostar and an antibody against intercellular adhesion molecule 1 (ICAM-1). Endostar is a modified version of recombinant human endostatin and has been approved by the State Food and Drug Administration for the treatment of non-small cell lung cancer in China. ICAM-1 is an endothelial adhesion molecule that is up-regulated at sites of atherosclerosis $(18,19)$. UTMD was performed to treat atherosclerotic plaque in an apolipoprotein $\mathrm{E}$ (APOE)-deficient mouse model of atherosclerosis.

\section{Materials and Methods}

Animal and atherosclerosis induction. Six wild-type and 27 apolipoprotein E-deficient (Apoe-/-) C57BL6 mice were obtained from Changzhou CAVENS Laboratory Animal Co., Ltd. (Changzhou, P.R. China). All mice were male, 3-4 weeks old and were maintained in a HEPA-filtered environment at $24-25^{\circ} \mathrm{C}$ and humidity at 50-60\%. The wild-type C57BL/6 mice were fed normal chow. The Apoe ${ }^{-l-}$ mice were fed a hypercholesterolemic diet (Opensource Animal Diets Co., Ltd., Changzhou, China) containing $1 \%$ cholesterol, $10 \%$ fat, $10 \%$ egg yolk power and $0.2 \%$ sodium cholate. All mice were maintained on their respective diets for 30 weeks. Three Apoe $^{-/-}$mice fed the hypercholesterolemic diet fed were then randomly selected and euthanized and their aortas processed for morphologic and histopathological examination in order to confirm development of atherosclerosis. Remaining animals were then treated as described below. Animal experiments were approved by the Animal Committee of Nanjing Origin Biosciences, P.R. China (OB 1607).

Microbubble preparation. Targestar-SA microbubble (Targeson Inc., San Diego, CA, USA; distributed in China by Origin Bioscience, Nanjing, P.R. China) was used as the basis for drug-loaded and targeted microbubbles in this study. Targestar-SA is an ultrasound contrast agent composed of a perfluorocarbon gas core encapsulated by a lipid shell. The outer shell is derivatized with streptavidin, which binds biotinylated ligands at a density of $80-220 \times 10^{3}$ molecules per microbubble. The agents have a median diameter of approximately $2.0 \mu \mathrm{m}$. Two types of microbubbles were produced.
ICAM1-targeted microbubbles (MBi) were prepared by incubating microbubbles with a biotinylated antibody to ICAM-1 (Abcam, Cambridge, MA, USA) at room temperature for $20 \mathrm{~min}$ at a ratio of 0.7 nmoles of the antibody per $10^{9}$ microbubbles. ICAM-targeted and Endostar-loaded therapeutic microbubbles (MBie) were prepared by coupling both biotinylated anti-ICAM-1 and Endostar (Simcere Pharmaceutical, Nanjing, P.R. China) to the outer shell of the same microbubble at a ratio of 0.35 nmoles of each targeting moiety per $10^{9}$ microbubbles. For fluorescence-activated cell sorting analysis of the prepared microbubbles, the biotinylated anti-ICAM1 and Endostar were labeled with fluorescent dyes fluorescein isothiocyanate and rhodamine, respectively. Unreacted anti-ICAM-1 and Endostar were removed from the microbubbles by centrifugal washing, per the manufacturer's recommended protocol. The presence and conjugation rate of anti-ICAM-1 and Endostar to the microbubble surface were examined by epifluorescence microscopy (Olympus BX53; Tokyo, Japan) and flow cytometry (BD Accuri C6). Microbubble-bound Endostar was measured by the BCA Protein Assay (Pierce, Rockford, IL, USA). Unconjugated TargestarSA microbubbles were used directly from the vial without the addition of ligand.

Treatment. Six wild-type C57BL/6 mice fed with normal chow were assigned to group 1 as normal control. The remaining 24 Apoe $^{-/-}$ mice were randomly divided into four groups of six mice and assigned to groups 2-5 after confirmation of atherosclerosis 30 weeks following a hypercholesterolemic diet. Group 2 mice were untreated and served as model control; group 3 mice received Endostar $(25 \mathrm{mg} / \mathrm{kg})$ by retro-orbital injection; group 4 mice received $\mathrm{MBi}$; group 5 mice received $\mathrm{MBie}$. Mice were anesthetized with ketamine, acepromazine and xylazine and then placed in a dorsal recumbency position on a heated stage. A dose of $50 \mu \mathrm{l}$ $\left(1 \times 10^{8}\right)$ microbubbles per mouse was administered by retro-orbital injection with a 27-gauge needle. UTMD was performed for groups 4 and 5 using a hand-held sonoporator (Sonitron 2000, Artison, OK, USA). This device consists of a piezoelectric transducer with a center frequency of $1 \mathrm{MHz}$. The transducer was placed above the ventral midline of the abdominal and thoracic cavities with ultrasound coupling gel. The settings were optimized as follows: duty cycle was $50 \%$, treatment duration of $30 \mathrm{~s}$, with acoustic intensity of $2 \mathrm{~W} / \mathrm{cm}^{2}$. The sonoporator cycled on and off in 30-s intervals to enable the microbubbles to fully re-perfuse the aortas after each 30-s cycle of UTMD. This process was repeated three times. All treatments and UTMD were repeated $48 \mathrm{~h}$ later with the same doses and settings.

Morphologic measurement. All animals were sacrificed 2 weeks after the final treatment. After euthanasia, mice were placed in dorsal recumbency, and a ventral midline incision was made to expose the abdominal and thoracic cavities. The aorta was perfused with phosphate-buffered saline (PBS) through the left ventricle of the heart. Next, under magnification, microscissors and fine-point forceps were used to remove the fat surrounding the three aortic arch branches (brachiocephalic, left common carotid, and left subclavian). The aorta was removed from its origin at the left ventricle to a few millimeters distal to its bifurcation into the common iliac arteries. The aorta was freed from surrounding vessels by making cuts at each of the three branches of the arch, the origin of the aorta at the heart, and distal to the aortic bifurcation into the common iliac arteries. The dissected aorta was then photographed. 

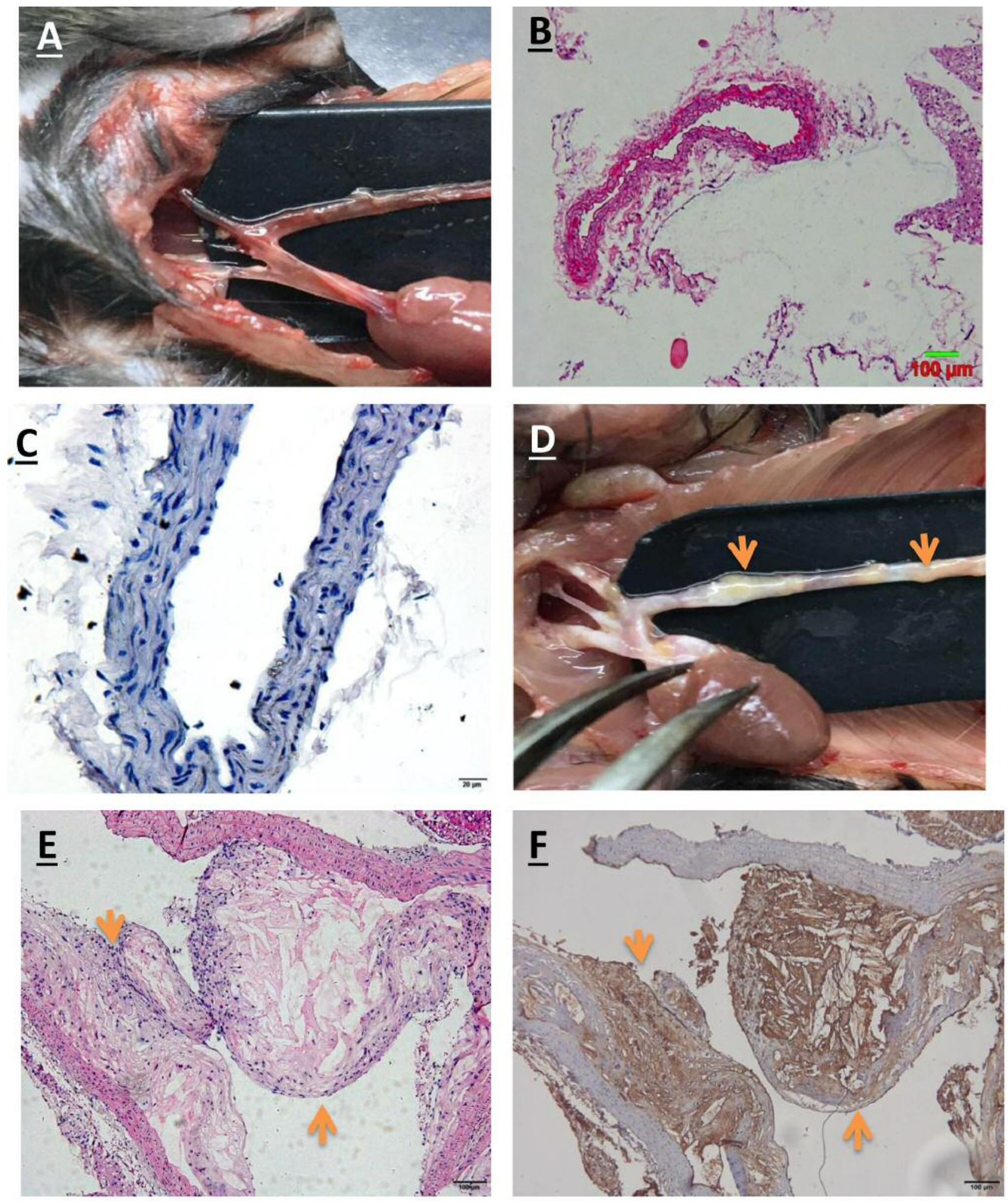

Figure 1. Atherosclerotic plaques in an apolipoprotein E (APOE)-deficient mouse model of atherosclerosis. Representative images for A: gross appearance, and B: hematoxylin and eosin $(H \& E)$ and $C$ : intercellular cell adhesion molecule-1 (ICAM-1) immunohistochemical staining of the aorta in normal control animals. Representative images for D: gross appearance, and E: H\&E and F: ICAM-1 immunohistochemical staining of the aorta with atherosclerotic plaque in an APOE-deficient mouse model of atherosclerosis. Orange arrows indicate atherosclerotic plaques. Magnification: H\&E 100x; immunohistochemical staining 400x. 

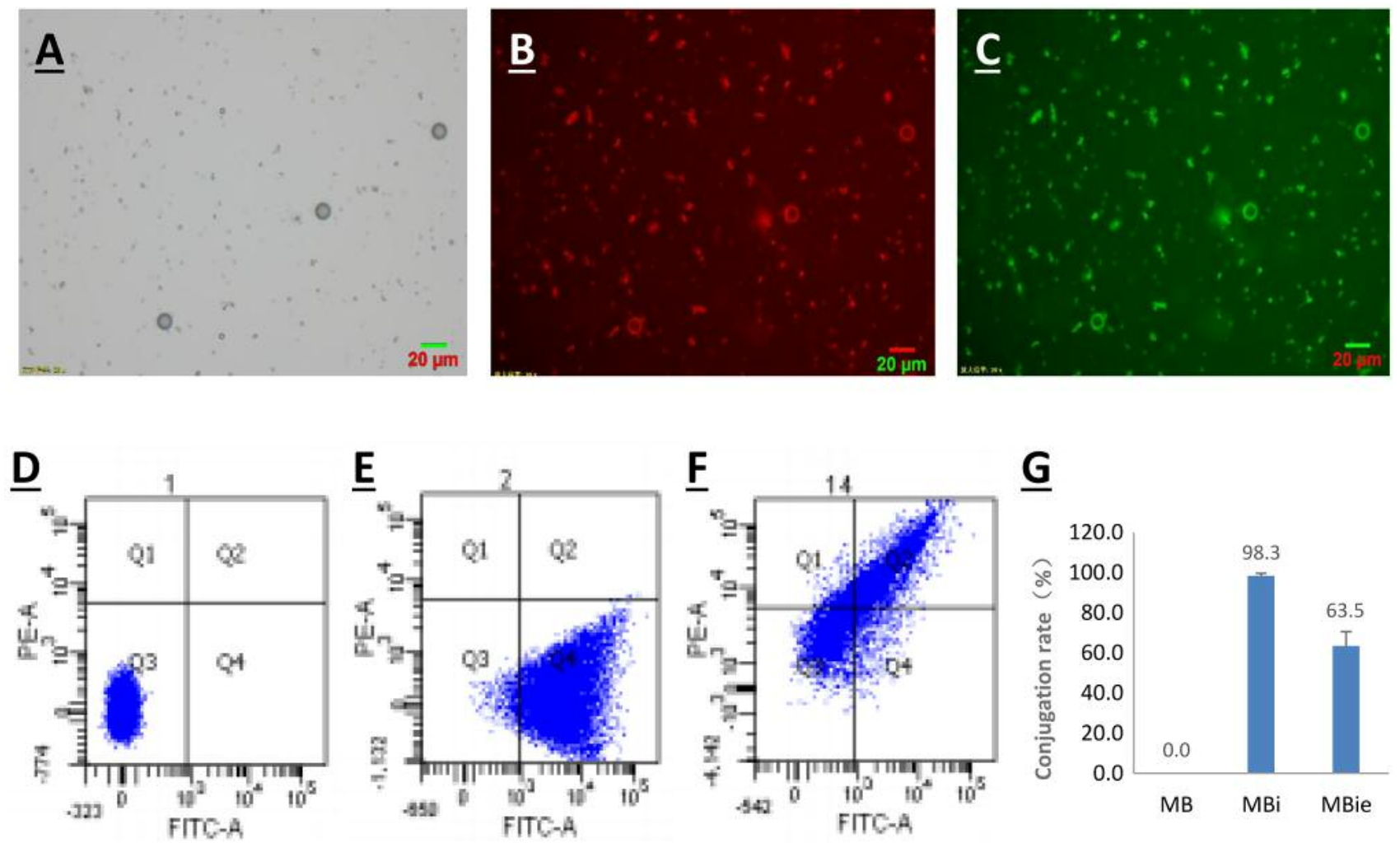

Figure 2. Microbubble charactarization. A: Representative bright-light image of microbubbles. B: Representative fluorescence image of the microbubbles conjugated with rhodamine-labeled Endostar. $C$ : Representative fluorescence image of microbubbles conjugated with fluorescein isothiocyanate-labeled antibody to intercellular cell adhesion molecule 1 (ICAM-1). Magnification: 400x. Flow cytometric analysis of: D: Unconjugated microbubbles (MB), E: ICAM-1-targeted microbubbles (MBi) and F: ICAM-1-targeted and endostar-loaded therapeutic microbubbles (MBie). G: Conjugation rate of MBi and MBie.

The areas of the entire dissected aorta and atherosclerotic plaques were measured with Image-Pro Plus 6.0 software (Media Cybernetics, Silver Spring, MD, USA). The percentage of en face plaque area was calculated for each animal.

Histology and immunohistochemistry. One segment of the aorta was obtained from each mouse after aorta perfusion. The aorta was fixed in $10 \%$ formalin, dehydrated, paraffin-embedded, sectioned at $4 \mu \mathrm{m}$ and stained with hematoxylin and eosin (H\&E). One microscopic slide with three sections from the paraffin block was created for each animal. Histological changes were evaluated by a pathologist who was blinded to the experimental groups. For immunohistochemical analysis, the sections were washed three times with PBS for $5 \mathrm{~min}$ each, blot dried and then treated with $3 \%$ hydrogen peroxide for 30 min at room temperature to block endogenous-peroxidase activity. The sections were immersed in antigen-retrieval solution (citrate buffer, $\mathrm{pH}$ 6.0) for $10 \mathrm{~min}$, followed by rinsing with PBS. After blocking with normal goat serum for $30 \mathrm{~min}$ at $37^{\circ} \mathrm{C}$, sections were co-incubated with primary antibodies against CD31 (BD Biosciences, San Diego, CA, USA) or ICAM-1 (Abcam) (1:100 dilution in PBS) overnight and then with a peroxidase-conjugated anti-rabbit IgG secondary antibody (Biosynthesis Biotechnology Co., Ltd., Beijing, China) for $1 \mathrm{~h}$ at room temperature. Subsequently, the sections were incubated with 3,3-diaminobenzidine reagent (ZSGB-BIO, Beijing, P.R. China) for $10 \mathrm{~min}$, counterstained with hematoxylin, dehydrated and mounted for microscopy. The slides were viewed at $100 \times$ or $400 \times$ magnification with brown-stained cells being identified as positivelystained cells. Expression levels were quantified by the average optical density (AOD) of the positively-stained cells in 5 fields/sample with Image-Pro Plus 6.0 software (Media Cybernetics).

Statistical analysis. Statistical analysis was performed using SPSS16.0 software (SPSS Inc., Chicago, IL, USA). All results are expressed as the mean \pm standard deviation (SD). Comparisons between two or multiple groups were made with the Student's $t$-test or ANOVA. Values of $p<0.05$ were considered significant.

\section{Results and Discussion}

Atherosclerotic plaque verification. As shown in Figure 1, lesions of atherosclerosis were grossly observed in the APOEdeficient mice 30 weeks following a hypercholesterolemic diet. The atherosclerotic plaques developed not only in the aortic root but throughout the aorta and its principal branches. The plaques were detected in the descending thoracic, lower 
A
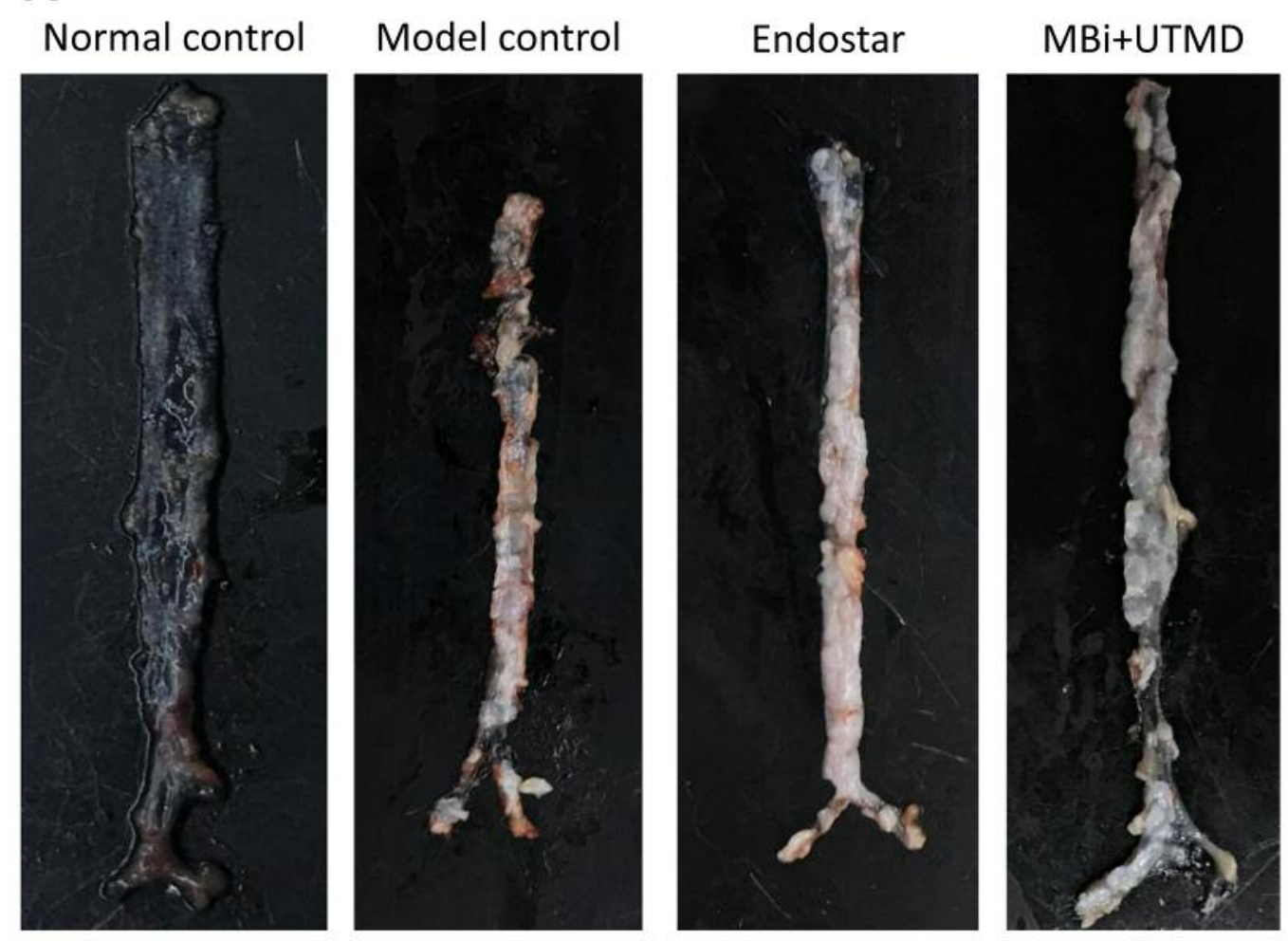

\section{MBie+UTMD}

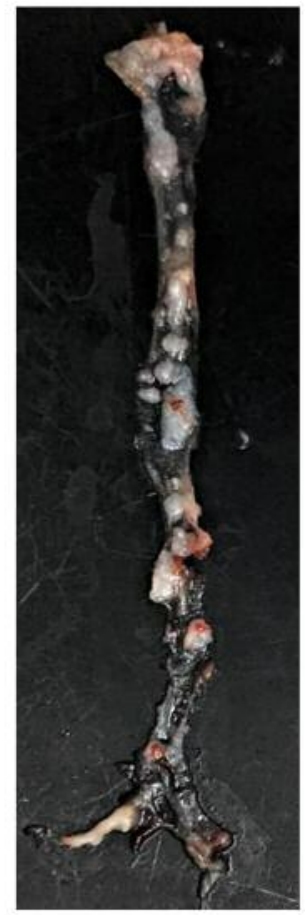

B

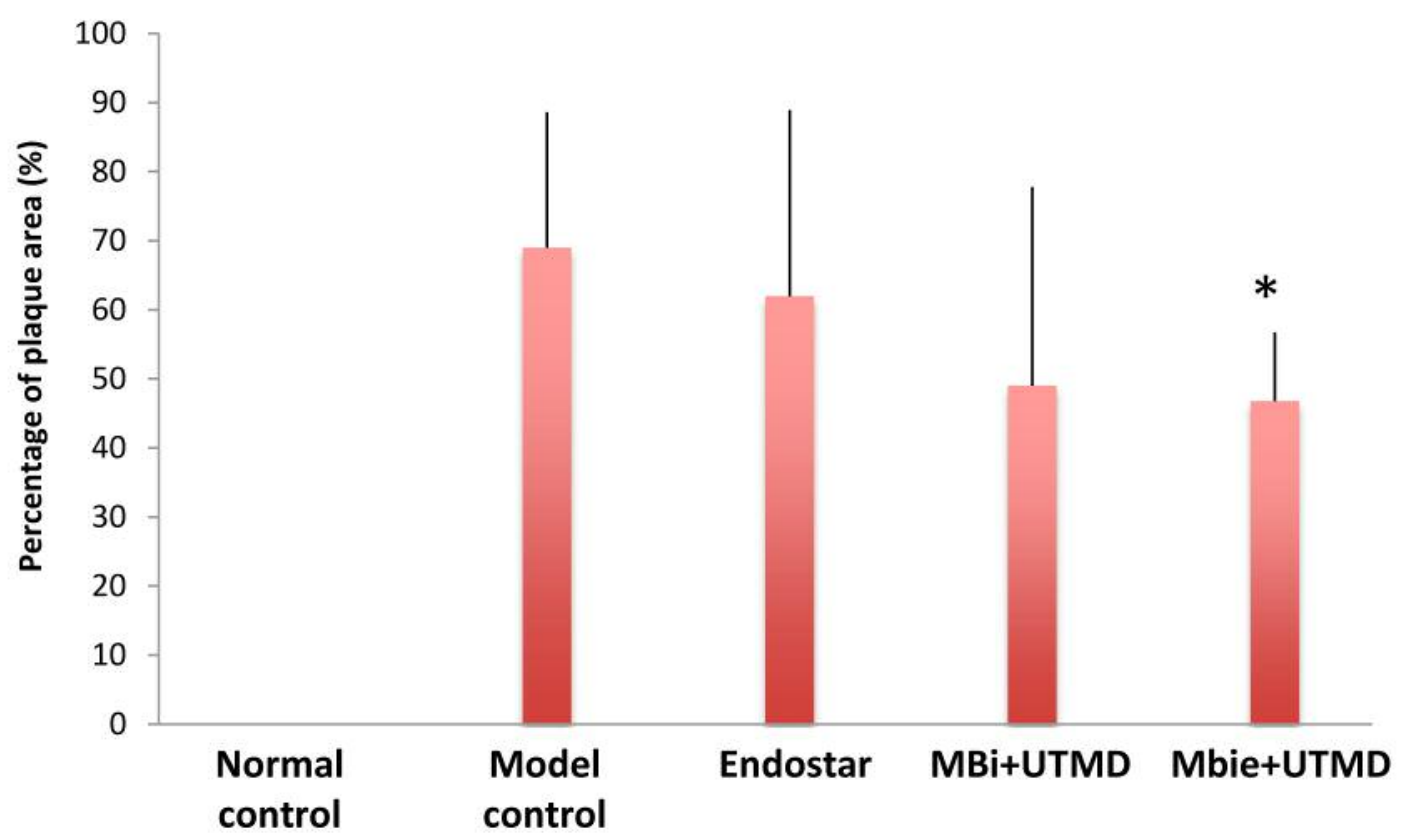

Figure 3. Efficacy of treatment in each group on atherosclerotic plaque progression in apolipoprotein E (APOE)-deficient mice. A: Representative images of atherosclerotic plaques in the aorta in each group. B: The percentage of en face plaque area in normal control, model control, and groups treated with intercellular cell adhesion molecule 1 (ICAM-1)-targeted microbubbles (MBi) with ultrasound-targeted microbubble delivery (UTMD), and ICAM1-targeted and Endostar-loaded therapeutic microbubbles (MBie) with UTMD. *Significantly different at p<0.05 compared to the model group. 

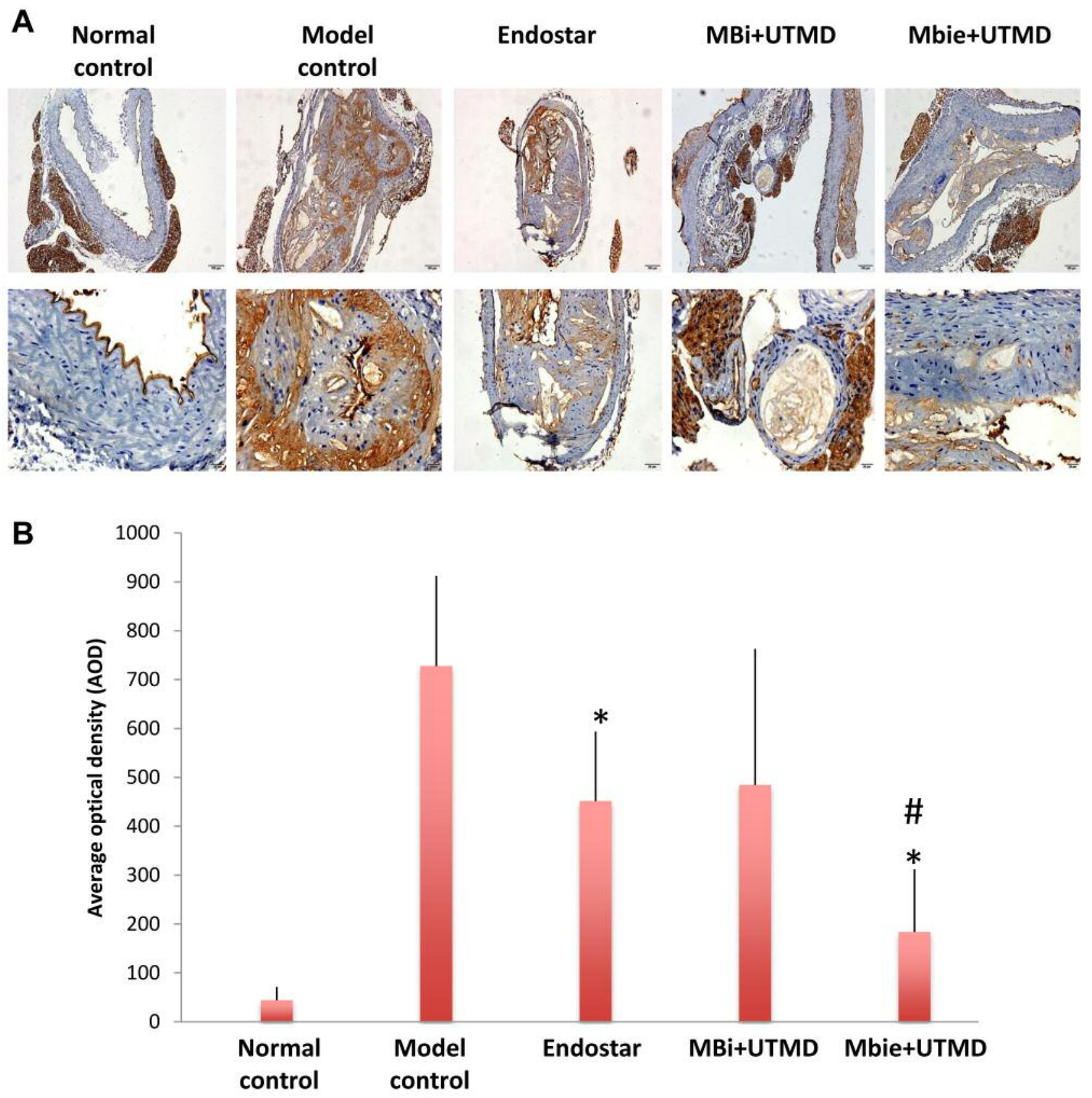

Figure 4. Efficacy of treatment in each group on expression of angiogenesis markers in atherosclerotic plaques. A: Representative immunohistochemical images for CD31 expression in normal control, model control, and groups treated with intercellular cell adhesion molecule 1 (ICAM-1)-targeted microbubbles (MBi) with ultrasound targeted microbubble delivery (UTMD), and ICAM-1-targeted and Endostar-loaded therapeutic microbubbles (MBie) with UTMD (magnification: upper panel 100x; lower panel 400x). B: Quantitation of the expression of CD31 by average optical density. Significantly different at $p<0.01$ compared with the *model control group, and "Endostar and MBi+UTMD groups.

abdominal, proximal coronary and common iliac arteries. Histological observations confirmed that fibrous plaques were present in APOE-deficient mice. These lesions contained necrotic cores together with a few foam cells that were covered by abundant fibrous tissue. Further immunohistochemical analysis demonstrated that the atherosclerotic plaques stained positively with the monoclonal antibody against ICAM-1, suggesting that ICAM-1 is expressed in atherosclerotic plaques. The normal-chow-fed wild-type mice did not exhibit any atherosclerotic plaques nor ICAM-1 expression. 
Microbubble characterization. Successful conjugation of ICAM-1 antibody and Endostar to the microbubbles was analyzed by epifluorescence microscopy and flow cytometry. As shown in Figure 2, fluorescently-labeled ICAM-1 antibody and Endostar were visualized on the surface of the microbubbles by epifluorescence microscopy. Flowcytometric analysis showed that the conjugation rate of $\mathrm{MBi}$ and MBie was $98.3 \%$ and $63.5 \%$, respectively. Quantitation of microbubble-bound Endostar by BCA Protein Assay revealed a maximum Endostar payload of $6 \mu \mathrm{g}$ per $10^{9}$ microbubbles.

UTMD with MBie microbubbles inhibited atherosclerotic plaque. To analyze the effects of UTMD on atherosclerotic plaque, the atherosclerotic lesions in the entire aorta of $\mathrm{Apoe}^{-/}$ mice were assessed in each experimental group. As shown in Figure 3, compared to the model control group, the animals in the group treated with UTMD with MBie had a significantly reduced plaque area $(46.78 \pm 9.98 \%$ versus $68.99 \pm 19.62 \%$, $p<0.05)$. There was no significant reduction of plaque area in groups treated with Endostar alone and UTMD with MBi as compared with the model control group, although reduced plaque area was observed in these two groups $(p>0.05)$.

UTMD with MBie microbubbles reduced expression of a marker of plaque angiogenesis. Immunohistochemical staining for angiogenesis marker CD31 was performed to assess the effect of the microbubbles on intraplaque neovascularization. As shown in Figure 4, high expression of $\mathrm{CD} 31$ was present in atherosclerotic plaque in the mouse model of atherosclerosis. The treatment with Endostar alone and UTMD with MBie significantly reduced CD31 expression as compared with the model control group $(p<0.01)$. Inhibition of CD31 expression was significantly greater in the group treated with UTMD with MBie as compared to those treated with Endostar alone and UTMD with MBi $(p<0.01)$. The group treated with UTMD with MBi did not show significantly lower CD31 expression as compared to the model control group $(p>0.05)$. This result indicates UTMD with MBie microbubbles had greater antiangiogenic efficacy against intraplaque neovascularization than Endostar alone and UTMD with MBi.

Intraplaque neovascularization plays a significant role in atherosclerotic plaque destabilization and rupture because of the additional supply of lipids and inflammatory mediators to the lesion. Considerable interest is focusing on a treatment approach targeting inhibition of microvessel formation and function within atherosclerotic plaques. Although some antiangiogenic therapies have been tested for atherosclerosis, only limited efficacy was observed (20). In the present study, we investigated a new strategy to treat athrosclerosis by UTMD targeting intraplaque neovascularization in an APOEdeficient mouse model of atherosclerosis. Therapeutic ultrasound using UTMD has demonstrated great potential as a tool for targeted disruption of tumor vasculature $(13,14)$. A particularly attractive strategy is loading of microbubbles with the therapeutic, thereby enabling local release of the payload upon destruction of microbubbles within the ultrasound-treated region (21-23). This technique is being explored for local gene transfection, targeted drug delivery and release, blood-brain barrier disruption and thrombolysis (24-26). However, to our knowledge, UTMD has never been used to treat atherosclerosis. In this study the microbubbles were conjugated with antibody to ICAM-1 as a targeting moiety and with Endostar as a therapeutic moiety. The study results demonstrated that UTMD with ICAM1-targeted and Endostar-loaded microbubbles (MBie) significantly reduced atherosclerotic plaque area and expression of intraplaque angiogenesis marker $\mathrm{CD} 31$.

Endostar, a novel recombinant human endostatin approved in China, inhibits tumor proliferation and metastasis as a strong inhibitor of angiogenesis (27). Endostar has been studied for inhibition and reduction of plaque neovascularization in animal models (28). In this study, we compared UTMD with ICAM-1-targeted and Endostar-loaded microbubbles (MBie group) with Endostar administration alone (Endostar group) and found UTMD with MBie microbubbles had greater inhibitory efficacy on atherosclerotic plaque and CD31 expression in the plaques. This indicates more efficient Endostar delivery and targeting with UTMD than mere intravascular administration of the drug.

In conclusion, UTMD targeting intraplaque neovascularization was found to inhibit atherosclerotic plaque in a mouse model of atherosclerosis. This suggests the potential of microbubble-mediated ultrasound technology in aiding drug therapy with improved drug delivery for atherosclerosis treatment.

\section{Conflicts of Interest}

None of the Authors have a conflict of interest in regard to this study.

\section{Acknowledgements}

This work was supported by the Hangzhou Science and Technology Development Fund 20150633B67.

\section{References}

1 Libby P: Inflammation and atherosclerosis. Circulation 105: 1135-1143, 2002.

2 Virmani R, Burke AP, Kolodgie FD and Farb A: Vulnerable plaque: the pathology of unstable coronary lesions. J Interv Cardiol 15: 439-446, 2002.

3 Carmeliet P: Angiogenesis in health and disease. Nat Med 9: 653-660, 2003. 
4 Moreno PR, Purushothaman KR, Fuster V, Echeverri D, Truszczynska H, Sharma SK, Badimon JJ and O'Connor WN: Plaque neovascularization is increased in ruptured atherosclerotic lesions of human aorta: Implications for plaque vulnerability. Circulation 110: 2032-2038, 2004.

5 Moulton KS: Plaque angiogenesis and atherosclerosis. Curr Atheroscler Rep 3: 225-233, 2001.

6 Fleiner M, Kummer M, Mirlacher M, Sauter G, Cathomas G, Krapf R and Biedermann BC: Arterial neovascularization and inflammation in vulnerable patients: early and late signs of symptomatic atherosclerosis. Circulation 110: 2843-2850, 2004.

7 Kolodgie FD, Gold HK, Burke AP, Fowler DR, Kruth HS, Weber DK, Farb A, Guerrero LJ, Hayase M, Kutys R, Narula J, Finn AV and Virmani R: Intraplaque hemorrhage and progression of coronary atheroma. N Engl J Med 349: 2316-2325, 2003.

8 Giannoni MF and Vicenzini E: Focus on the "unstable" carotid plaque: detection of intraplaque angiogenesis with contrast ultrasound. Present state and future perspectives. Curr Vasc Pharmacol 7: 180-184, 2009.

9 Virmani R, Kolodgie FD, Burke AP, Finn AV, Gold HK, Tulenko TN, Wrenn SP and Narula J: Atherosclerotic plaque progression and vulnerability to rupture: angiogenesis as a source of intraplaque hemorrhage. Arterioscler Thromb Vasc Biol 25: 2054-2061, 2005

10 Stefanadis C, Toutouzas K, Stefanadi E, Lazaris A, Patsouris E and Kipshidze N: Inhibition of plaque neovascularization and intimal hyperplasia by specific targeting vascular endothelial growth factor with bevacizumab-eluting stent: an experimental study. Atherosclerosis 195: 269-276, 2007.

11 Bot I, Jukema JW, Lankhuizen IM, van Berkel TJ and Biessen EA: Atorvastatin inhibits plaque development and adventitial neovascularization in APOE-deficient mice independent of plasma cholesterol levels. Atherosclerosis 214: 295-300, 2011.

12 Kuhnast, S, van der Hoorn JW, van den Hoek AM, Havekes LM, Liau G, Jukema JW and Princen HM: Aliskiren inhibits atherosclerosis development and improves plaque stability in APOE*3Leiden.CETP transgenic mice with or without treatment with atorvastatin. J Hypertens 30: 107-116, 2012.

13 Smith AH, Fujii H, Kuliszewski MA and Leong-Poi H: Contrast ultrasound and targeted microbubbles: diagnostic and therapeutic applications for angiogenesis. J Cardiovasc Transl Res 4: 404415, 2011

14 Zhang C, Huang PT, Zhang Y, Chen J, Shentu WH, Sun Y, Yang ZJ and Chen SY: Anti-tumor efficacy of ultrasonic cavitation is potentiated by concurrent delivery of anti-angiogenic drug in colon cancer. Cancer Letters 347: 105-113, 2014.

15 Tlaxca JL, Anderson CR, Klibanov AL, Lowrey B, Hossack JA, Alexander JS, Lawrence MB and Rychak JJ: Analysis of in vitro transfection by sonoporation using cationic and neutral microbubbles. Ultrasound Med Biol 36: 1907-1918, 2010.

16 Bekeredjian R, Chen S, Frenkel PA, Grayburn PA and Shohet RV: Ultrasound-targeted microbubble destruction can repeatedly direct highly specific plasmid expression to the heart. Circulation 108: 1022-1026, 2003.
17 Kinoshita M and Hynynen K: Intracellular delivery of Bak BH3 peptide by microbubble enhanced ultrasound. Pharm Res 22: 716-720, 2005.

18 Galkina E and Ley K: Vascular adhesion molecules in atherosclerosis. Arterioscler Thromb Vasc Biol 27: 2292-2301, 2007.

19 Bourdillon MC, Poston RN, Covacho C, Chignier E, Bricca G and McGregor JL: ICAM1 deficiency reduces atherosclerotic lesions in double-knockout mice $\left(\right.$ Apoe $\left.\left.^{(-/-}\right) / \operatorname{Icam1}\left(^{-/-}\right)\right)$fed a fat or a chow diet. Arterioscler Thromb Vasc Biol 20: 26302635,2000

20 Van der Veken B, De Meyer GR and Martinet W: Intraplaque neovascularization as novel therapeutic target in advanced atherosclerosis. Expert Opin Ther Targets 20: 1247-1257, 2016.

21 Bazan-Peregrino M and Arvanitis CD, Rifai B, Seymour LW and Coussios CC: Ultrasound-induced cavitation enhances the delivery and therapeutic efficacy of an oncolytic virus in an in vitro model. J Controlled Release 157: 235-242, 2012.

22 Arvanitis CD, Bazan-Peregrino M, Rifai B, Seymour LW and Coussios CC: Cavitation-enhanced extravasation for drug delivery. Ultrasound Med Biol 37: 1838-1852, 2011.

23 Wang JF, Wu CJ, Zhang CM, Qiu QY and Zheng M: Ultrasoundmediated microbubble destruction facilitates gene transfection in rat C6 glioma cells. Mol Biol Rep 36: 1263-1267, 2009.

24 Dittmar KM, Xi J, Hunter F, Trimble C, Bur M, Frenkel V and Li KC: Pulsed high-intensity focused ultrasound enhances systemic administration of naked DNA in squamous cell carcinoma model: Initial experience. Radiology 235: 541-546, 2005.

25 Mizushige K, Kondo I, Ohmori K, Hirao K and Matsuo H: Enhancement of ultrasound-accelerated thrombolysis by echo contrast agents: dependence on microbubble structure. Ultrasound Med Biol 25: 1431-1437, 1999.

26 Bekeredjian R, Grayburn PA and Shohet RV: Augmentation of cardiac protein delivery using ultrasound targeted microbubble destruction. Ultrasound Med Biol 31: 687-691, 2005.

27 Ling Y, Yang Y, Lu N, You QD, Wang S, Gao Y, Chen Y and Guo QL: Endostar, a novel recombinant human endostatin, exerts antiangiogenic effect via blocking VEGF-induced tyrosine phosphorylation of KDR/FLK-1 of endothelial cells. Biochem Biophys Res Commun 361: 79-84, 2007.

28 Mao W, Kong J, Dai J, Huang ZQ, Wang DZ, Ni GB and Chen ML: Evaluation of recombinant endostatin in the treatment of atherosclerotic plaques and neovascularization in rabbits. $\mathrm{J}$ Zhejiang Univ Sci B 11: 599-607, 2010.
Received May 15, 2018

Revised June 21, 2018

Accepted June 28, 2018 